$$
\begin{aligned}
\left\{[\alpha 0]^{2} a^{-1}+[\beta 0]^{2} b^{-1}+\cdots\right\}\left\{\alpha[0]^{2} a^{-2}+[\beta 0]^{2} b^{-2}\right. & +\cdots\}^{-1 / 2} \\
& =\left\{[\alpha 0]^{2}+[\beta 0]^{2}+\cdots\right\}^{1 / 2},
\end{aligned}
$$

and this is satisfied when the factors $a, b, c, \cdots$ are all equal. Without loss of generality, they may be taken equal to unity. For this weighting system, the formation of the damped normal equations (10) may be thought of as being accomplished simply by the addition of a positive constant, $1 / w$, to the coefficients of the principal diagonal of the standard normal equations (5). Another weighting system which has been used successfully is, $a=[\alpha \alpha], b=[\beta \beta], \cdots$; in this case the damped normal equations are formed by multiplying the principal diagonal coefficients of the standard normal equations by a constant greater than unity, $1+1 / w$.

The nature of the damping which we have imposed upon the parameter variables can be given a simple geometric interpretation. For instance, if the unity weighting system is considered, the "overshooting" of the solution is prevented by damping the distance ( $k$ dimensional) from the initial solution point, since $Q$ is then the square of this distance. By this restriction of $k$ dimensional distance (which would appear to be a natural way to prevent overshooting), we are not obliged to decide on an arbitrary preassigned procedure restricting the variables individually, as is done, for example, by the method of Cauchy (1.c.). The greater freedom given the individual variables by the method of damped least squares may account for the fact that it has solved, with a comparatively rapid rate of convergence, types of problems which are of much greater complexity than those to which the principle of least squares is ordinarily applied.

\title{
ON THE DEFLECTION OF A CANTILEVER BEAM*
}

By H. J. BARTEN (Washington Navy Yard)

In spring theory it is sometimes necessary to compute the deflection of a cantilever beam for which the squares of the first derivatives cannot be neglected as is done in classical beam theory. This problem is thus placed in the same category as the problem of the elastica.

The solution given in this note can be applied to a cantilever of any stiffncss. The difference between the deflection as found by the classical beam theory and that found by the present method is, however, noticeable only in the case of beams of low stiffness.

The clamped end of the beam is taken as the origin of coordinates and downward deflections are considered as positive. A point on the beam may be identified by four quantities of which only one is independent. These four quantities are the two rectangular coordinates $x$ and $y$, the arc length $s$ measured from the origin of coordinates, and the deflection angle $\theta$ which is the angle between the tangent to the curve at the point under discussion and the horizontal. We may thus identify this point by the symbol $(x, y, s, \theta)$. The subscript $L$ is used to identify the value of these quantities at the free end of the beam. Before deflection a vertical load $P$ is applied at the point $(L, 0, L, 0)$. The beam has a uniform cross section of moment of inertia $I$ and is com-

* Received Feb. 21, 1944. 
posed of a material whose modulus of elasticity is $E$. The problem is to find the deflection of the end-point of the beam due to the vertical load $P$.

The bending moment induced at the point $(x, y, s, \theta)$ by the vertical load $P$ is

$$
M=P\left(x_{L}-x\right) .
$$

Therefore

$$
d \theta / d s=a\left(x_{L}-x\right),
$$

where $a=P / E I$. Using the relation

we obtain

$$
\frac{d \theta}{d s}=\frac{d \theta}{d x} \frac{d x}{d s}=\cos \theta \frac{d \theta}{d x},
$$

$$
\int \cos \theta d \theta=\int a\left(x_{L}-x\right) d x
$$

or

$$
\sin \theta=a\left(x_{L} x-\frac{1}{2} x^{2}\right)+C .
$$

The boundary condition at the clamped end of the beam, namely, $\theta=0^{-}$when $x=0$, reduces Eq. (2) to

$$
\sin \theta=a\left(x_{L} x-\frac{1}{2} x^{2}\right) .
$$

Thus

$$
\sin \theta_{L}=\frac{1}{2} a x_{L}^{2} .
$$

Combining the latter expression and Eq. (3) we obtain

$$
\sin \theta_{L}-\sin \theta=\frac{1}{2} a\left(x_{L}-x\right)^{2} .
$$

Thus

$$
x_{L}-x=\left[2 a^{-1}\left(\sin \theta_{L}-\sin \theta\right)\right]^{1 / 2} .
$$

Substituting this expression into Eq. (1), we obtain

or

$$
\frac{d \theta}{d s}=\frac{d \theta}{d y} \frac{d y}{d s}=\sin \theta \frac{d \theta}{d y}=\left[2 a\left(\sin \theta_{L}-\sin \theta\right)\right]^{1 / 2},
$$

Therefore

$$
y=\int_{0}^{\theta} \frac{\sin \theta d \theta}{\left[2 a\left(\sin \theta_{L}-\sin \theta\right)\right]^{1 / 2}} .
$$

$$
y_{L}=\int_{0}^{\theta_{L}} \frac{\sin \theta d \theta}{\left[2 a\left(\sin \theta_{L}-\sin \theta\right)\right]^{1 / 2}} .
$$

With the transformation

$$
\cos \left(\frac{\pi}{4}-\frac{\theta}{2}\right)=\cos \left(\frac{\pi}{4}-\frac{\theta_{L}}{2}\right) \sin \phi=k \sin \phi,
$$

Eq. (6) becomes

where

$$
y_{L}=a^{-1 / 2} \int_{\delta}^{\pi / 2} \frac{\left(2 k^{2} \sin ^{2} \phi-1\right) d \phi}{\left(1-k^{2} \sin ^{2} \phi\right)^{1 / 2}}
$$




$$
\sin \delta=\frac{\cos \pi / 4}{k}, \quad k=\cos \left(\frac{\pi}{4}-\frac{\theta_{L}}{2}\right) .
$$

Eq. (7) is a combination of incomplete and complete elliptic integrals ${ }^{1}$ and may be written

$$
y_{L}=a^{-1 / 2}[F(k)-F(k, \delta)-2 E(k)+2 E(k, \delta)],
$$

where $F(k)$ and $E(k)$ are the first and second complete elliptic integrals respectively and $F(k, \delta)$ and $E(k, \delta)$ are the first and second incomplete elliptic integrals respectively.

As Eq. (8) stands it is useless unless we find $\theta_{L}$ as a function of $a$ and $L$. This relationship may be obtained in the following manner. From Eq. (1) we get

$$
\theta_{L}=\int_{0}^{L} a\left(x_{L}-x\right) d s
$$

Integrating by parts we obtain

$$
\theta_{L}=\int_{0}^{x_{L}} a s d x=\int_{0}^{L} a s \frac{d x}{d s} d s=\int_{0}^{L} a s \cos \theta d s .
$$

Differentiating this latter integral with respect to its upper limit, we have

$$
d \theta_{L} / d L=a L \cos \theta_{L} .
$$

The solution to this differential equation is

$$
\sin \theta_{L}=\tanh \frac{a L^{2}}{2} .
$$

'This completes the solution to the problem.

In order to compare our results with those of Gross and Lehr ${ }^{2}$ we must express our solution in the same dimensionless factors that they employed. By dividing the actual deflection of the beam by the "small deflection" $a L^{3} / 3$ they obtain a deflection factor which is a function of the dimensionless quantity $a L^{2}$. We shall call this deflection factor $F_{y}$. Thus, from Eq. (8)

$$
F_{y}=\frac{3 y_{L}}{a L^{3}}=3\left(a L^{2}\right)^{-3 / 2}[F(k)-F(k, \delta)-2 E(k)+2 E(k, \delta)] .
$$

In order to find the maximum bending stress at the clamped end of the beam we must know the length of the moment arm $x_{L}$. Combining Eqs. (4) and (9) we find that

$$
x_{L}^{2}=\frac{2}{a} \tanh \frac{a L^{2}}{2} .
$$

Gross and Lehr use the dimensionless contraction factor $x_{L} / L$ an an aid in finding $x_{L}$. We shall define this factor as $F_{x}$. Thus

${ }^{1}$ Jahnke and Emde, Funktionentafeln mit Formeln und Kurven, Dover Publications, 1943.

${ }^{2}$ Gross and Lehr, Die Federn, V:D. I. Verlag, 1938. 


$$
F_{x}^{2}=\frac{2}{a L^{2}} \tanh \frac{a L^{2}}{2} .
$$

Computations show that Gross and Lehr's values of $F_{y}$ have a constantly increasing error which deviates about $4 \%$ from our results when $a L^{2}=1$.

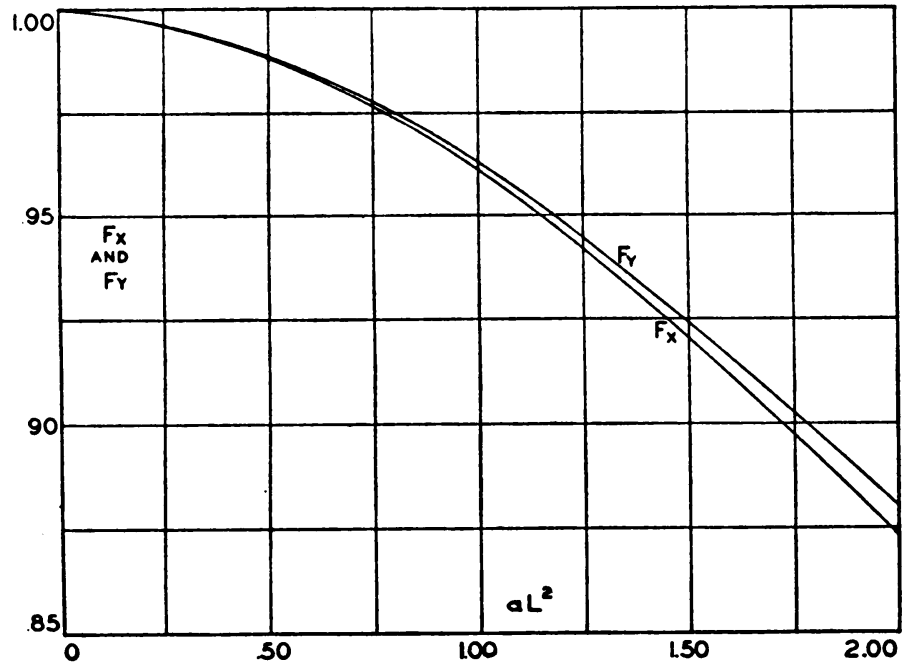

Fig. 1.

The two factors $F_{x}$ and $F_{y}$ are very important to the designer. For this reason curves of these two factors with $a L^{2}$ as the independent variable are given in Fig. 1. The values of $F_{y}$ were computed from Jahnke and Emde.

\section{ON WAVES IN BENT PIPES*}

\section{By S. $\Lambda$. SCHELKUNOFF (Bell Telephone Laboratories)}

In a recent issue of this QUARTERLY, ${ }^{1}$ Karlem Riess obtained expressions for the fields of electromagnetic waves in bent pipes of rectangular cross section by the perturbation method. While it is true that in a bent pipe the waves cannot be classified into transverse electric and transverse magnetic types because in general both $E$ and $H$ have components in the direction of wave propagation, a different classification into two types is possible. This permits another method which yields the general solution in terms of Bessel functions.

In the one wave type, the plane of the electric ellipse is normal to the axis of bending (the $Y$-axis in Figure 1, p. 329 of Riess' paper); these waves have been called electrically oriented $\left(E O_{m, n}\right.$ wave type) and the fields of these waves are obtainable from $H_{y}$ which may be expressed as the product of Bessel and sine (or cosine) functions.

* Received Feb. 18, 1944.

1 Vol. 1, No. 4, pp. 328-333. 